\title{
Polarisation to colour transformation via nano- antenna enhanced quantum dot emission
}

\author{
T.D. James, E. Panchenko, T.L. Nguyen, P. Mulvaney, T.J. Davis, A. Roberts
}

Abstract- Metallic nano-antennas have been shown to be an excellent candidate for enhancing and directing optical emission from semiconductor based quantum dots (QD). QD photoluminescence (PL) enhancement is achieved by placing a suitably orientated QD in the near-field of a resonant metallic nano-antenna. Through the careful design of an optical Vee-antenna, two distinct visible resonances can be obtained, enabling the polarization dependant enhancement of two different QD sources, thus producing a polarization-tocolour transformation at the nano-scale. Possible future applications include an optical nano-scale demultiplexer device.

Index Terms-Plasmon, Quantum dots, Antennas, Photoluminescence

\section{INTRODUCTION}

Enhancement of quantum sources has been a hot topic of research in the plasmonics community for a number of years. One of the main drivers of this research effort has been the desire to create a single-photon source at the nano-scale, which has applications in photon based computing and quantum cryptography [1]. Much of the work on plasmonics based single photon sources involves creating an optical nano-antenna with desirable properties, such as directionality for the Yagi-Uda antenna [2], and placing either lithographically or via a pick-and-place method a single photon source in the desired optimal location near the plasmonic antenna [3]. These studies have produced enhanced quantum sources on the nano-scale, however, they are limited in their functionality to only exciting or switching a single source. This work aims to produce a plasmonic nanoantenna device that can selectively excite two separate quantum sources, where each source can be discriminated through the polarisation of the excitation source. This polarization selective device could form the central component of a nano-scale optical demultiplexer in a quantum computing/communications system, which is able to

T.D. James, E. Panchenko and A. Roberts are with the School of Physics at the University of Melbourne, Parkville, Victoria, Australia 3010 (e-mail: timothy.james@unimelb.edu.au).

T.D. James and T.J. Davis are with the Melbourne Centre for Nanofabrication (MCN), Australian National Fabrication Facility, Clayton, Victoria, 3168, Australia

T.J. Davis is with the Commonwealth Scientific and Industrial Research Organisation (CSIRO), Materials Science and Engineering, Private Bag 33, Clayton South MDC, Victoria, 3169, Australia

T.L. Nguyen and P. Mulvaney are with Bio21 Institute and School of Chemistry, The University of Melbourne, Parkville, Victoria 3010 Australia
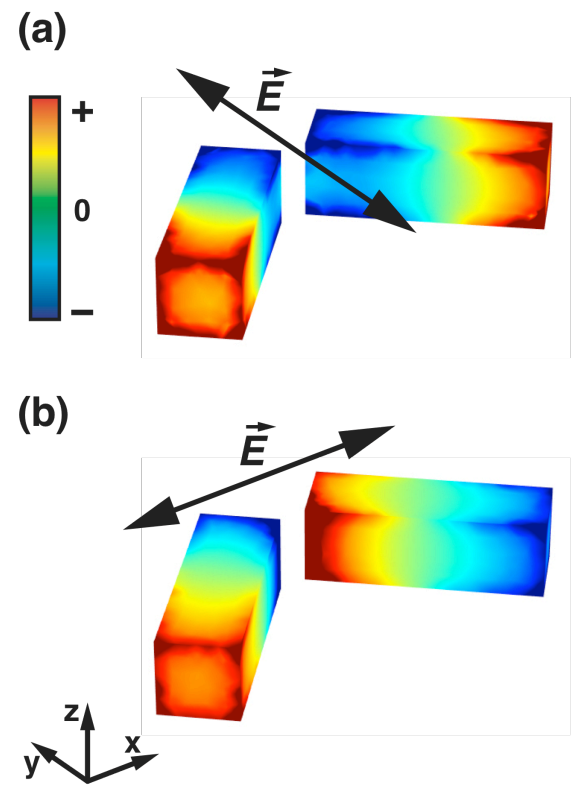

Fig. 1. Surface charge of the Vee antenna at resonance when excited with (a) $\mathrm{y}$ and (b) x polarised light.

transform a polarisation-controlled input signal to the discrete wavelengths of the enhanced quantum sources.

\section{TwO SOURCE ENHANCEMENT}

To achieve two-source enhancement with a plasmonic nano-antenna, a number of design constraints must be met. The PL maxima of the two sources must be known such that the two resonances of the nano-antenna can match and enable the enhancement of the PL. This work uses $\mathrm{CdSe} / \mathrm{ZnS}$ based QDs that can have a PL emission range of approximately 500-700nm, depending on the size of the QD.

Previously, we studied a range of RF-inspired plasmonic antennas for PL enhancement, specifically the J-pole and Vee antenna families [4]. The AWX and J-pole antennas display two polarization-depenant resonant modes. They are, however, spectrally too far apart to fit within the spectral range of $\mathrm{CdSe} / \mathrm{ZnS}$ QDs. The Vee antenna on the other hand appears to be an excellent candidate as the two resonances for the orthogonal polarizations are spectrally close enough together to fit the QD spectral range and tunable by varying the antenna's design parameters. Fig. 1 illustrates the surface charge on the antenna for the two orthogonal resonant modes.

In order to combine the QDs with the Vee antenna nanostructures, QDs are dispersed in an epoxy/photoresist SU8 


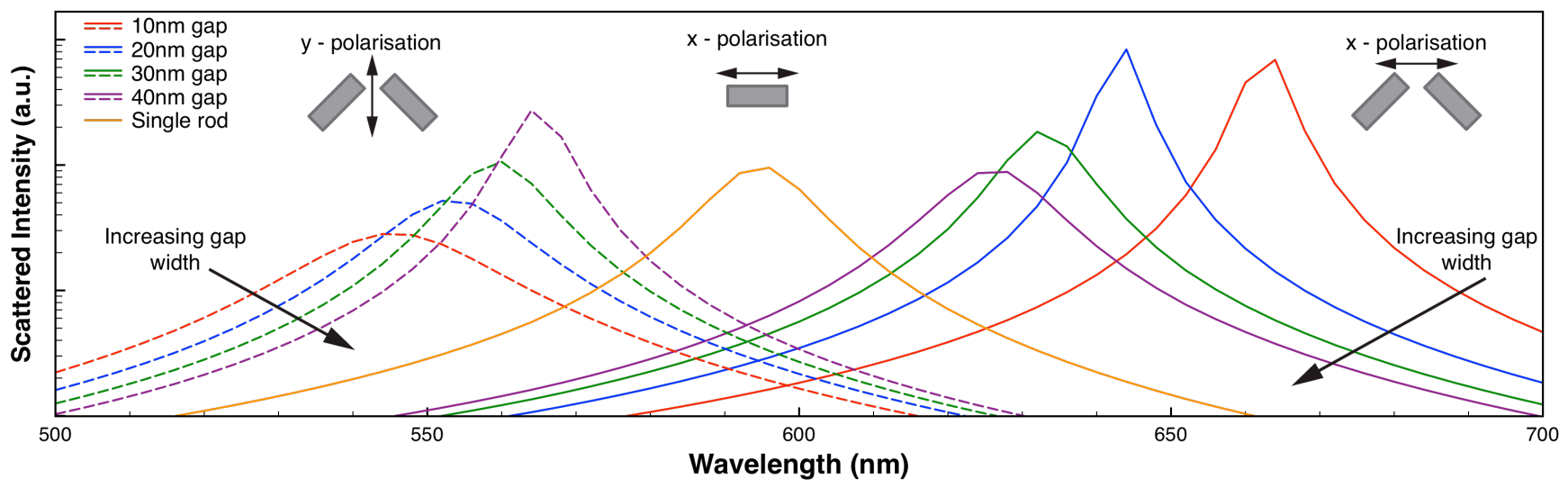

Fig. 1. Scattering spectra for Al Vee antennas with different gap widths between the two antenna arms. The Vee antennas are excited by both $\mathrm{x}$ and $\mathrm{y}$ polarised light to reveal two resonant modes, where the single antenna arm is excited along its long axis.

2000.5, commonly used in Micro Electrical Mechanical Systems (MEMS) as a structural material, and spun onto the glass substrate/antenna surface. Coating the nano-antennas with a polymer, such as SU8, increases the refractive index of the medium surrounding the antennas, which results in a significant red-shifting in the wavelength of the resonant modes. To reduce the wavelength of the resonant mode back to the desired spectral position, the antenna size must be reduced or a different material with a lower plasma frequency must be considered. Significantly reducing the size of the Vee antenna structures from the published values [4] would require significant effort in terms of fabrication, yet altering the material from $\mathrm{Ag}$ to $\mathrm{Al}$ is easily achievable. Comparatively $\mathrm{Ag}$ is a superior plasmonic material due to its lower losses, however $\mathrm{Ag}$ oxidises readily in atmosphere. This means that the inevitable delays between fabrication, characterisation and QD coating can occur with plasmonic devices will not affect the optical properties of $\mathrm{Al}$ antennas.

Taking the design constraints of a high refractive index dielectric environment and the spectral range restrictions of the $\mathrm{CdSe} / \mathrm{ZnS}$ QDs, an Al based Vee antenna design was found to be suitable. The dimensions of each arm of the Vee antenna are $30 \mathrm{~nm}$ width, $90 \mathrm{~nm}$ length, $30 \mathrm{~nm}$ thick orientated at 90 degrees to each other, where the gap between the two arms is varied. Fig. 2 shows modeling results obtained with COMSOL Multi-Physics 4.4, which uses the Finite Element Method to determine the scattering properties of the plasmonic nano-antennas. It is clear from this study that reducing the gap between the two arms of the Vee antenna results in a widening of the spectral gap of the two orthogonal resonances. For reference, a single arm of the Vee antenna was simulated, which shows that as the two arms of the antenna are moved apart, the interaction between them is reduced, resulting in the resonances converging on the single arm resonance.
These simulation results strongly indicate that the Vee antenna is an excellent candidate for two-source enhancement of $\mathrm{CdSe} / \mathrm{ZnS}$ QDs, as the design fits the desired spectral range, and can be tuned to across the spectrum to achieve enhancement of QDs of nearly any size. An experimental investigation into the polarisation dependant excitation of two distinct quantum sources by Vee antennas will be presented, along with a study into optimising the optical properties of the Vee antenna structures.

\section{ACKNOWLEDGMENT}

This research was supported under the Australian Research Council's Discovery Projects funding scheme (project number DP110100221), and through the Melbourne Centre for Nanofabrication Technical Fellowship program, and the University of Melbourne Early Career Researcher Grant Scheme. This work was performed in part at the Melbourne Centre for Nanofabrication (MCN) in the Victorian Node of the Australian National Fabrication Facility (ANFF)

\section{REFERENCES}

1 Choy, J.T., Bulu, I., Hausmann, B.J.M., Janitz, E., Huang, I.-C., and Loncar, M.: 'Spontaneous emission and collection efficiency enhancement of single emitters in diamond via plasmonic cavities and gratings', Applied Physics Letters, 2013, 103, (16), pp. 161101-161101

2 Curto, A.G., Volpe, G., Taminiau, T.H., Kreuzer, M.P., Quidant, R., and van Hulst, N.F.: 'Unidirectional Emission of a Quantum Dot Coupled to a Nanoantenna', Science, 2010, 329, (5994), pp. 930-933

3 Gruber, C., Kusar, P., Hohenau, A., and Krenn, J.R.: 'Controlled addressing of quantum dots by nanowire plasmons', Applied Physics Letters, 2012, 100, (23), pp. 231102-231102-231103

4 James, T.D., Davis, T.J., and Roberts, A.: 'Optical investigation of the J-pole and Vee antenna families', Optics Express, 2014, 22, (2), pp. 1336-1341 